\title{
Clinical utility of mifepristone over oxytocin in preventing adversities of parturition outcomes: a comparative study
}

\author{
Mudita Jain ${ }^{1}$, Rituja Kaushal2* \\ ${ }^{1}$ Department of Obstetrics and Gynecology, ${ }^{2}$ Department of Community Medicine, LNMC and RC, Bhopal, Madhya
} Pradesh, India

Received: 20 October 2018

Accepted: 25 October 2018

*Correspondence:

Dr. Rituja Kaushal,

E-mail: dr.rituja@gmail.com

Copyright: $\odot$ the author(s), publisher and licensee Medip Academy. This is an open-access article distributed under the terms of the Creative Commons Attribution Non-Commercial License, which permits unrestricted non-commercial use, distribution, and reproduction in any medium, provided the original work is properly cited.

\begin{abstract}
Background: Of the various medical methods of induction, induction with oxytocin and prostaglandins remain the most popular and acceptable methods in modern obstetric practice. The present cross-sectional study conducted in the Department of Obstetrics and Gynecology, Kamla Raja Hospital, Gwalior, mifepristone has been used through oral route for induction of labor. The objectives of the present study were to evaluate the effect of oral mifepristone for induction of labor, to record the outcome of labor and the incidence of operative interference, and to see any adverse effects on mother and/or neonate with its use, to compare its effect with other medical method of labor induction.

Methods: The present study is a prospective comparative study carried out in the Department of Obstetrics and Gynecology, G. R. Medical College and Kamla Raja Hospital, Gwalior (M.P.), from May 2009 to June 2010. Total number of patients involved in the study is 119 , study group comprised of 69 patients in which oral mifepristone $(200 \mathrm{mg}$ ) was given on day 1 and day 2 of a four-day observation period. The control group comprised of 50 patients induced with intravenous oxytocin group.

Results: On overall assessment of the efficacy of labour induction with oral mifepristone as compared to intravenous (I/V) oxytocin, we found that there was no significant difference in the mode of delivery (vaginal and caesarean section) and Apgar score.

Conclusions: The induction of active labour induction, induction to delivery interval is higher in mifepristone group as compared to oxytocin group. However, the drug resulted in higher rates of vaginal birth after cesarean section (VBAC) with no grave maternal and fetal outcomes, so thus aspect of oral mifepristone is of great consideration and requires further research.
\end{abstract}

Keywords: Comparative study, Delivery outcome, Mifepristone, Oxytocin, Tertiary care centre

\section{INTRODUCTION}

Giving birth to a baby on one hand provides happiness to the mother and entire family, but on the other hand it has always been darkened with pain, agony and fear of some mishap, more common with the first confinement. For ages long, there has been no solution for the problems of prolonged labour and non-progress of labour leading ultimately to great mortality and disability from infections and operations. With the passage of time, attention is now been focused for a healthy pregnancy outcome, even if it means to initiate labour earlier than it would take place as a natural event. ${ }^{1,2}$

The aim of successful induction is to achieve vaginal delivery when continuation of pregnancy presents a threat to the life or wellbeing of the mother or her unborn child. The infant should be delivered in good condition within 
an acceptable time frame and with a minimum of maternal discomfort or side effects. While inducing labour, the obstetrician is attempting to induce prematurely the two interlinked components of labour: cervical ripening and uterine contractility.

The objective of the pharmacological induction of a physiological process is an attempt to mimic the natural process as closely as possible. Even today the means available are not regarded as specific, as no method is certain of success and none is entirely free from risk. Of the various medical methods of induction, induction with oxytocin and prostaglandins remain the most popular and acceptable methods in modern obstetric practice.

The present study conducted in the Department of Obstetrics and Gynecology, Kamla Raja Hospital, Gwalior, mifepristone has been used through oral route for induction of labor.

The objectives of the present study were to evaluate the effect of oral mifepristone for induction of labor, to record the outcome of labor and the incidence of operative interference, to see any adverse effects on mother and/or neonate with its use, to compare its effect with other medical method of labor induction.

\section{METHODS}

The present study is a prospective comparative study carried out in the Department of Obstetrics and Gynecology, G.R. Medical College and Kamla Raja Hospital, Gwalior (M.P.), from May 2009 to June 2010. Total number of patients involved in the study is 119 , study group comprised of 69 patients in which oral mifepristone $(200 \mathrm{mg}$ ) was given on day 1 and day 2 of a four-day observation period. The control group comprised of 50 patients induced with intravenous oxytocin group.

\section{Inclusion criteria}

- Patients with pregnancy duration between 36-42 weeks, age 18-35 years, singleton pregnancy with cephalic presentation and were indicated for labor induction for one or more of the following medical/obstetric reasons were included in the study like PIH (pregnancy induced hypertension), postdated, oligohydraminos, term pregnancy, IUGR (intra uterine growth retardation), prev.

\section{Exclusion criteria}

- LSCS, no contraindication for vaginal delivery and the patients of known haemoglobinopathies, placenta previa, transverse lie or presentation other than cephalic, cephalo-pelvic disproportion, unexplained vaginal bleeding, history of previous difficult or traumatic labor.
Initial assessment of patients was done as per routine and Bishop Score was noted, after that the patient was given oral preparation of mifepristone $(200 \mathrm{mg})$ on day 1 and day 2 of a four-day observation period, time of insertion of drug was noted in each case, they were assessed at 24hour interval. Meticulous maternal and fetal monitoring followed this.

At regular intervals their vital signs, time of onset of uterine contractions and relaxation, and fetal heart rate was charted and per vaginum examination was repeated as and required to note the progress of labor. Trial interruption followed by active intervention was done whenever there was any sign of fetal distress, unnoticed CPD, cervical dystocia, maternal exhaustion, in coordinate uterine activity.

The mode of delivery was noted which could be either a vaginal delivery or caesarean section. Special attention was given to note the state of lower uterine segment in caesarean section as regards increased vascularity, atonicity after extraction of baby, post-partum hemorrhage and the methods to obviate it.

In cases of vaginal delivery, careful watch was done to note postpartum hemorrhage either due to atonicity or due to traumatic vaginal delivery, or to traumatic vaginal delivery, or simply to note increased vascularity state of cervix.

The fetal outcome in terms of date, time of delivery, sex and weight of baby, presence of meconium stained liquor, live or still birth was recorded.

The mother and child were followed up for a period of at least 72 hours for postpartum hemorrhage, infection in mother and meconium aspiration syndrome in child.

\section{RESULTS}

In study group mean gestation period is 39.3 and in control group mean gestation period is 40.2 , both are comparable (Table 1).

Table 1: Distribution of study population in frequency numbers and percentage as per period of gestation.

\begin{tabular}{|lllll|}
\hline \multirow{2}{*}{ Gestation in weeks } & Case & \multicolumn{3}{c|}{ Control } \\
& No. & $\%$ & No. & \% \\
\hline $37-38$ & 22 & 31.9 & 8 & 16 \\
\hline $39-40$ & 12 & 17.4 & 17 & 34 \\
\hline$>40$ & 35 & 50.7 & 25 & 50 \\
\hline Total & 69 & 100 & 50 & 100 \\
\hline
\end{tabular}

Indication for induction remains same in both groups, Maximum number of cases belongs to postdate in both study $(43.5 \%)$ and control group (42\%), followed by term $(21.7 \%)$ in study group and $(32 \%) \mathrm{PIH}$ and $(20 \%)$ term patients in control group (Table 2). 
Table 2: Distribution of study population in frequency numbers and percentage as per indication for induction.

\begin{tabular}{|lllll|}
\hline Indication & Case & \multicolumn{3}{c}{ Control } \\
\hline PIH & 6 & 8.71 & 16 & 32 \\
\hline Postdate & 30 & 43.5 & 22 & 42 \\
\hline PROM & 0 & 0 & 2 & 04 \\
\hline Term & 15 & 21.7 & 10 & 20 \\
\hline Prev LSCS & 12 & 17.4 & 0 & 0 \\
\hline Oligo & 6 & 8.7 & 0 & 0 \\
\hline
\end{tabular}

Table 3 represents distribution of cases on basis of bishop score before induction, maximum number of patients in study group belong to bishop score (1-3), mean bishop score 1.92 and in control group maximum number of cases belong to bishop score (2-4), mean bishop score 2.9.

Table 3: Distribution of study population in frequency numbers and percentage as per nulliparous status and pre-induction Bishop score.

\begin{tabular}{|lll|ll|}
\hline Bishop score & Study & \multicolumn{2}{c|}{ Control } \\
\hline 1 & No. & \% & No. & \% \\
\hline 2 & 15 & 21.7 & 1 & 2 \\
\hline 3 & 12 & 17.4 & 9 & 18 \\
\hline 4 & 12 & 17.4 & 11 & 22 \\
\hline 5 & 0 & - & 5 & 10 \\
\hline
\end{tabular}

Table 4 represents distribution of cases on basis of bishop score before induction, in study group maximum number of cases belong to bishop score $3(21.7 \%)$ and bishop score $2(13 \%)$, mean bishop score 2.6 and in control group max. number of cases belong to bishop score 2 (20\%) and bishop score 3 (14\%), mean bishop score 2.8 .

Table 4: Distribution of study population in frequency numbers and percentage as per p1-p3 status and preinduction bishop score.

\begin{tabular}{|lllll|}
\hline Bishop score & Case & \multicolumn{3}{c|}{ Control } \\
\hline 1 & No. & $\%$ & No. & $\%$ \\
\hline 2 & 3 & 4.35 & - & - \\
\hline 3 & 9 & 13 & 10 & 20 \\
\hline 4 & 15 & 21.7 & 7 & 14 \\
\hline 5 & 3 & 4.35 & 4 & 8 \\
\hline
\end{tabular}

Table 5 represents distribution of cases on basis of interval from induction $o$ to active labor i.e. with favorable bishop score. The mean interval period in study group is 33.036 hours and mean interval in control group is 17.7 hours, $\mathrm{P}$ value $<0.002$, highly significant.

Table 6 represents distribution of cases on basis of interval from induction to active labor i.e. with favorable bishop score. The mean interval period in study group is 27.37 hours and mean interval in control group is 18.3 hours, $\mathrm{P}$ value $<.048$ is significant.

Table 5: Distribution of study population in frequency numbers and percentage as per nulliparous status for induction to active labor interval.

\begin{tabular}{|lllll|}
\hline Time & Case & \multicolumn{3}{c|}{ Control } \\
\hline No hours & 3 & $\%$ & No. & $\%$ \\
\hline 6-12 hours & 9 & 13 & 5 & 10 \\
\hline 13-24 hours & 6 & 8.7 & 10 & 20 \\
\hline 25-48 hours & 6 & 8.7 & 7 & 14 \\
\hline$>$ 48 hours & 15 & 21.7 & - & - \\
\hline
\end{tabular}

Table 6: Distribution of study population in frequency numbers and percentage as per p1-p3 status for induction to active labor interval.

\begin{tabular}{|lllll|}
\hline \multirow{2}{*}{ Time } & Case & \multicolumn{3}{c|}{ Control } \\
\hline$<6$ hours & No. & $\%$ & No. & $\%$ \\
\hline 6-12 hours & 3 & 4.35 & 5 & 10 \\
\hline 13-24 hours & 9 & 13 & 3 & 6 \\
\hline 25-48 hours & 3 & 13 & 8 & 16 \\
\hline$>$ 48 hours & 6 & 4.35 & 6 & 12 \\
\hline
\end{tabular}

Table 7 represents distribution of cases on basis of interval from ingestion of drug to expulsion of baby, either vaginally or by caesarean section.

The mean interval period in study group is 45 hours and mean interval in control group is 19.8 duration in hours, $\mathrm{P}$ value $<0.001$ is highly significant.

Table 7: Distribution of study population in frequency numbers and percentage as per nulliparous status and application to expulsion interval.

\begin{tabular}{|c|c|c|c|c|}
\hline \multirow{2}{*}{ Time } & \multicolumn{2}{|c|}{ Case } & \multicolumn{2}{|c|}{ Control } \\
\hline & No. & $\%$ & No. & $\%$ \\
\hline$<6$ hours & 0 & $0 \%$ & 2 & 4 \\
\hline 6-12 hours & 6 & 15.4 & 7 & 14 \\
\hline 13-24 hours & 6 & 15.4 & 11 & 22 \\
\hline $25-48$ hours & 10 & 25.6 & 8 & 16 \\
\hline$>48$ hours & 17 & 43.6 & 0 & 0 \\
\hline
\end{tabular}

Table 8 represents distribution of cases, on basis of interval from ingestion of drug to expulsion of baby, either vaginally or by caesarean section. The mean interval period in study group is 36.4 hours and mean interval in control group is 21.9 duration in hours, $\mathrm{P}$ value $<0.006$ is highly significant.

Table 9 represents distribution of cases on basis of outcome. Cases having vaginal delivery (including VBAC) in study group $(78.3 \%)$ and in control group $(72 \%)$. 
Table 8: Distribution of study population in frequency numbers and percentage as per p1-p3 status and application to expulsion interval.

\begin{tabular}{|lllll|}
\hline Time & Study & \multicolumn{3}{c|}{ Control } \\
\hline <6 hours & No. & $\mathbf{\%}$ & No. & $\%$ \\
\hline 6-12 hours & 0 & 0 & 5 & 10 \\
\hline 13-24 hours & 3 & 10 & 0 & 0 \\
\hline 25-48 hours & 10 & 33.3 & 8 & 16 \\
\hline$>$ 48 hours & 7 & 33.3 & 9 & 18 \\
\hline
\end{tabular}

Cases undergoing caesarean section in study group $(21.7 \%)$ and in control group $(28 \%)$ ( RR $0.75, \mathrm{P}$ value $<0.02$ ), there was not much difference in both group in terms for outcome as total vaginal delivery or caesarean section, however VBAC $9(13 \%)$ cases contribute an important part in study group as compare to control group.

Table 9: Distribution of study population in frequency numbers and percentage as per outcome/ mode of delivery.

\begin{tabular}{|lllll|}
\hline \multirow{2}{*}{ Mode of delivery } & Study & \multicolumn{3}{c|}{ Control } \\
& No. & $\%$ & No. & $\%$ \\
\hline Vaginal delivery & 45 & 65.2 & 36 & 72 \\
\hline VBAC & 9 & 13 & 0 & - \\
\hline LSCS & 15 & 21.7 & 14 & 28 \\
\hline
\end{tabular}

Table 10 represents distribution of cases on basis of indication for cesarean section, in study group maximum number of cases $(13 \%)$ is due to fetal distress and in control group (14\%) is due to fetal distress. No cases in study group was noted due to failed induction as compare to control group (8\%) cases was noted due to failed induction.

Table 10: Distribution of study population in frequency numbers and percentage as per indication for cesarean section.

\begin{tabular}{|lll|ll|}
\hline \multirow{2}{*}{ Indication for LSCS } & Case & & \multicolumn{2}{c|}{ Control } \\
\cline { 2 - 6 } & No. & $\%$ & No. & $\%$ \\
\hline FD & 9 & 13 & 7 & 14 \\
\hline NPOL & 3 & 4.34 & 3 & 6 \\
\hline Failed induction & 0 & - & 4 & 8 \\
\hline DTA & 0 & - & 0 & - \\
\hline TMSL & 3 & 4.34 & 0 & - \\
\hline
\end{tabular}

No maternal complication seen in study group, whereas in control group there was increased risk of PPH and uterine tachysystole. $\mathrm{P}$ value $<0.009$, is highly significant.

Table 12 shows distribution of cases, on basis of fetal complication, Maximum number of cases due to meconium stain in study group is $(4.34 \%)$ which is comparable with control group $(4 \%)$, however in control group max. number of cases was due to respiratory distress (24\%), (RR 0.13, P <0.009).

Table 11: Distribution of study population in frequency numbers and percentage as per maternal complications.

\begin{tabular}{|lllll|}
\hline \multirow{2}{*}{ Complication } & Study & \multicolumn{2}{c|}{ Control } \\
\hline PPH & No. & $\%$ & No. & $\%$ \\
\hline Dt Cx tear & 0 & - & 5 & 10 \\
\hline$\uparrow L U S$ vascularity & 0 & - & 2 & 4 \\
\hline$\uparrow C x$ vascularity & 0 & - & 0 & - \\
\hline Atonicity & 0 & - & 0 & - \\
\hline Uterine tachysytole & 0 & - & 3 & 6 \\
\hline
\end{tabular}

Table 12: Distribution of study population in frequency numbers and percentage as per fetal complications.

\begin{tabular}{|lllll|}
\hline \multirow{2}{*}{ Complication } & Case & \multicolumn{2}{c|}{ Control } \\
\hline Meconium stain & 3 & 4.34 & 2 & 4 \\
\hline MAS & 0 & - & 0 & - \\
\hline RD & 0 & - & 12 & 24 \\
\hline Delayed cry & 0 & - & 1 & 2 \\
\hline
\end{tabular}

\section{DISCUSSION}

In the present study entitled "Prospective comparative study for induction of labor by mifepristone and its comparison with oxytocin" was carried out on patients with pregnancy duration between $37-42$ weeks. The pregnancy was singleton with cephalic presentation. The study was carried out from May 2009 to July 2010. Depending upon the agent used for induction of labour (mifepristone versus oxytocin), patients were divided into two groups; Study and Control respectively. 69 patients with indications for labour induction were induced with $200 \mathrm{mg}$ of mifepristone. The study was compared with a Control group of 50 patients, induced with intravenous oxytocin. The patients selected for labour induction ranged from nullipara to third para. The maximum number of patients were nullipara in both study and control groups. Labour was induced in patients having period of gestation ranging from 37 weeks to more than 40 weeks, maximum number patients in study groups belongs to $>40$ weeks of gestation. The average gestation in study group is 39.3 . In $50 \%$ of patients of control group the period of gestation is $>40$ weeks, the average being 40.2 weeks, which is comparable with those of study group. More than $60 \%$ of patients in study group and control group belongs to post date or term pregnancy. The other indications being PIH, pregnancy with previous caesarean section, oligohydramnios, preterm rupture of membranes. Successful labour induction depends upon whether the cervix is ripe or not prior to induction. Cervical ripening can be best stated by doing preinduction Bishop Score, with unfavorable cervix, the 
mean duration from start of induction to delivery increases. The study group induced with oral mifepristone, the nulliparous study group, and $34.8 \%$ of patients had Bishop score 2 or 3 , and $21.7 \%$ of patients had Bishop score 1, mean Bishop Score 1.92. As compared to nulliparous patients, patients in $\mathrm{P} 1-\mathrm{P} 3$ group had slightly more favorable Bishop score, $34.7 \%$ of patients had Bishop score 2 or 3, Mean Bishop score 2.6, and overall Bishop score in the study group was <4. These results of ours were comparable to other studies. ${ }^{1-5}$

Induction to active labour interval in present study had an average duration of 33.036 hours in nulliparous patients as compared to 27.37 hours in P1-P3 groups. As compared to control the difference is highly significant $(\mathrm{p}<0.002)$ in nullipara patients $(17.7$ hours $)$ and $(\mathrm{p}<0.048)$ in multiparous patients (18.3 hours). In nullipara group, $26.05 \%$ patients had favorable Bishop Score within 24 hours as compared to control (42\%). Relative risk is 0.61 , $\mathrm{p}$ value is (NS). In multipara patients $30.4 \%$ patients had favorable Bishop Score in < 24 hours as compared to $32 \%$ in control group. Relative risk is 0.95 , p value (NS). The average time from application to expulsion interval was 45 hours in nulliparous as compared to 19.8 hours in control group. $\mathrm{p}$ value $<0.001$ which is highly significant. The average time from application to expulsion interval in multiparous patients was 36.4 hours as compared to 21.9 hours in control groups. $\mathrm{p}$ value $<0.006$ which is also significant. Our findings resembled with findings suggested by Stenlund and Wing.,

In nulliparous study groups $17.4 \%$ of patients induced with oral mifepristone delivered within 24 hours as compared to $40 \%$ of patients in control group. Relative risk is 0.42 . In multiparous study group $18.84 \%$ of patients induced with oral mifepristone delivered within 24 hours as compared to $26 \%$ of patients in control group, relative risk is 0.72 . The results (mifepristone induced patients delivered within 24 hours) is compared with different studies which compared labour induction with mifepristone versus other method for labour induction or with placebo. ${ }^{6,7}$ In the study group, as per mode of delivery 54 patients delivered vaginally $(78.2 \%)$ out of which 9 patients $(13 \%)$ are VBAC, 15 patients $(21.7 \%)$ had caesarean section (RR- 0.75 , p value $<0.02$ ). Results are comparable with control group 36 patients $(72 \%)$ delivered vaginally and 14 patients $(28 \%)$ had caesarean section. The indication for which operative delivery was performed was fetal distress in both groups. However, no case was noted due to failed induction in mifepristone groups as compared to $4(8 \%)$ cases in control group. However, the difference was not statistically significant as compared to control.

The results were compared with different studies which compared labour induction with mifepristone versus other method for labour induction or with placebo for vaginal delivery. ${ }^{4,5,8}$ The result was compared with different studies which compared labour induction with mifepristone versus other method for labour induction or with placebo for operative interference. ${ }^{1-4,6,9,10}$

In the study group, amazingly no maternal complication was seen as compared to control group where 5 cases (10\%) had PPH and 2 cases (4\%) had tachysystole. Only 3 cases of fetal complications were noted $(4.34 \%)$ for meconium stained baby but no baby developed MAS as compared to control 2 cases $(4 \%)$ were meconium stain. On the contrary, respiratory distress was high (24\%) in control group and no case seen in study group. Overall fetal complication relative risk comes out to be 0.13 with significant $\mathrm{p}$ value, so this association is not by chance. The mean Apgar score at $1 \mathrm{~min}$ and at $5 \mathrm{~min}$ in mifepristone group is 7.94 and 9.46 as compared to oxytocin group (7.8 at $1 \mathrm{~min}$ and 8.2 at $5 \mathrm{~min}$ ). On overall assessment of the efficacy of labour induction with oral mifepristone as compared to I / V oxytocin, we found that there was no significant difference in the mode of delivery (vaginal and caesarean section) and Apgar score.

The induction of active labour induction, induction to delivery interval is higher in mifepristone group as compared to oxytocin group. However, the drug resulted in higher rates of VBAC with no grave maternal and fetal outcomes, so thus aspect of oral mifepristone is of great consideration and requires further research.

\section{CONCLUSION}

On overall assessment of the efficacy of labour induction with oral mifepristone as compared to intravenous oxytocin, we found that there was no significant difference in the mode of delivery (vaginal and caesarean section) and Apgar score. The induction of active labour induction, induction to delivery interval is higher in mifepristone group as compared to oxytocin group. However, the drug resulted in higher rates of VBAC with no grave maternal and fetal outcomes, so thus aspect of oral mifepristone is of great consideration and requires further research.

\section{Funding: No funding sources}

Conflict of interest: None declared

Ethical approval: The study was approved by the Institutional Ethics Committee

\section{REFERENCES}

1. Lelaidier C, Baton C, Benifla JL, Fernandez H, Bourget P, Frydman R. Mifepristone for labour induction after previous caesarean section. Randomized controlled trial. Br J Obstet Gynaecol. 1994;101(6):501-3.

2. Giacalone PL, Targosz V, Laffargue F, Boog G, Faure JM. Cervical ripening with mifepristone before labor induction: a randomized study. Randomized controlled trial. Obstet Gynecol. 1998;92(4 Pt 1):487-92. 
3. Elliott CL, Brennand JE, Calder AA. The effects of mifepristone on cervical ripening and labor induction in primigravidae. Randomized controlled trial. Obstet Gynecol. 1998;92(5):804-9.

4. Stenlund PM, Ekman G, Aedo AR, Bygdeman M. Induction of labor with mifepristone--a randomized, double-blind study versus placebo. Randomized controlled trial. Acta Obstet Gynecol Scand. 1999;78(9):793-8.

5. Gallot D, de Lapasse C, Houlle C, Sapin V, Laurichesse-Delmas H, Saulnier JP, et al. Obstetrical prognosis of labour induction with mifepristone after 41 weeks of gestation. Gynecol Obstet Fertil. 2004;32(9):708-12.

6. Wing DA, Fassett MJ, Mishell DR. Mifepristone for preinduction cervical ripening beyond 41 weeks' gestation: a randomized controlled trial. Randomized controlled trial. Obstet Gynecol. 2000;96(4):543-8.

7. Wing DA, Guberman C, Fassett M. A randomized comparison of oral mifepristone to intravenous oxytocin for labor induction in women with prelabor rupture of membranes beyond 36 weeks' gestation.
Randomized controlled trial. Am J Obstet Gynecol. $2005 ; 192(2): 445-51$.

8. Cabrol D, Dubois C, Cronje H, Gonnet JM, Guillot M, Maria B, et al. Induction of labor with mifepristone (RU 486) in intrauterine fetal death. Clinical Trial. Am J Obstet Gynecol. 1990;163(2):540-2.

9. Su H, Li E, Weng L. Mifepristone for induction of labor. Randomized controlled trial. Zhonghua $\mathrm{Fu}$ Chan Ke Za Zhi. 1996;31(11):676-80.

10. Berkane N, Verstraete L, Uzan S, Boog G, Maria B. Use of mifepristone to ripen the cervix and induce labor in term pregnancies. Randomized controlled trial. Am J Obstet Gynecol. 2005;192(1):114-20.

Cite this article as: Jain M, Kaushal R. Clinical utility of mifepristone over oxytocin in preventing adversities of parturition outcomes: a comparative study. Int J Reprod Contracept Obstet Gynecol 2018;7:4808-13. 